\title{
Co-morbidity between diabetes, migraine and depression
}

\author{
Line Iden Berge ${ }^{1,2,3}$, Trond Riise ${ }^{3}$ and Marjolein Iversen ${ }^{4,5}$ \\ 1) Department of Clinical Medicine, Section of Psychiatry, Faculty of Medicine and Dentistry, \\ University of Bergen, Norway \\ 2) Mood Net Research Group, Division of Psychiatry, Haukeland University Hospital, Bergen, Norway \\ 3) Research group of Lifestyle Epidemiology, Department of Public Health and Primary Health Care, \\ Faculty of Medicine and Dentistry, University of Bergen, Norway \\ 4) Centre for Evidence-Based Practice, Bergen University College, Bergen, Norway \\ 5) Department of Medicine, Section of Endocrinology, Stavanger University Hospital, Stavanger, Norway \\ Correspondence: Line Iden Berge, Institutt for samfunnsmedisinske fag, Kalfarveien 31, 5018 Bergen \\ E-mail: line.iden.berge@gmail.com Telephone: +4755586100
}

\begin{abstract}
Background: Young persons using oral antidiabetic agents have increased prevalence of medically treated migraine and depression. In the general population there is a well-known association between migraine and depression. Using medication as a proxy for disease, we investigated to which extent the previously reported increased prevalence of migraine in young persons using oral antidiabetic agents could be explained by co-morbid depression.

Methods: Data on all individuals in Norway aged 20-39 years being prescribed anti-diabetic $(\mathrm{n}=5055)$, migraine $(\mathrm{n}=26058)$ and/or antidepressant agents $(\mathrm{n}=57424)$ in 2006 was obtained from the Norwegian Prescription Database and analysed in a cross-sectional design using logistic regression.

Results: Persons using oral antidiabetic agents but no antidepressant agents had an overall increased sex and age adjusted OR of using migraine agents compared to persons not using oral anti-diabetic agents $(\mathrm{OR}=1.48 ; 95 \% \mathrm{CI}: 1.26,1.74)$. Persons using antidiabetic and antidepressant agents also had an increased $\mathrm{OR}$ of receiving migraine agents $(\mathrm{OR}=1.37 ; 1.03,1.82)$. Estimating these risks for men and women separately, no major differences were found.

Conclusion: The findings in the present study give no support for the hypothesis that the increased prevalence of migraine in young persons treated with oral antidiabetic agents partly could be explained by co-morbid depression.
\end{abstract}

\section{BACKGROUND}

Persons with diabetes are at increased risk of several complications and chronic diseases (1), and recent years it has been increasing interest in studying psychiatric and neurological diseases in relation to diabetes. Crosssectional and prospective studies have demonstrated that patients with type 2 diabetes have increased prevalence of co-morbid depression (2), and that they also have increased risk of developing depression over time, and vice versa, suggesting a bi-directional association between the two conditions (3). Further, both clinical and epidemiological studies have suggested that patients suffering from both diabetes and depression have poorer self-reported health than persons suffering from several other chronic diseases (4), poorer compliance regarding medication and dietary regimen (5), higher mean $\mathrm{HbAlc}(6)$, increased risk of diabetes related complications (7) as well as increased mortality $(8,9)$. The increased risk for, as well as the impact of, depression in patients with diabetes has led the American Diabetes Association to recommend screening for depression, anxiety and psychosocial problems as an ongoing part of the follow-up treatment of diabetes (10).

In the general population there is a well established association between depression and migraine (11). However, less is known about the frequency of migraine in patients with diabetes. One populationbased cross sectional study using data from the HUNT 2 Survey found that the prevalence of migraine among patients with diabetes varied according to age, with higher prevalence in lower age groups and lower prevalence in higher age groups (12). We have recently verified these results using medication as a proxy for disease. We found that persons less than 40 years of age who used oral antidiabetic medication had an increased prevalence of using migraine agents (13).

In the same study population we have also shown that persons treated with oral antidiabetic agents have an increased prevalence of using antidepressant agents, with the highest OR ranging from about 2 to 4.5 in the age-group 20-39 years (14). Because of the known association between depression and migraine in the general population (11), we wanted to study whether the previously reported increased prevalence of migraine in young persons treated with oral antidiabetic agents partly could be explained by co-morbid depression.

\section{METHODS}

The study population consisted of the complete Norwegian population aged 20 to 39 years by January 1, $2006(\mathrm{n}=1255353$, males $\mathrm{n}=635625$ and females $\mathrm{n}=$ 619 728). All individuals in this population who had 
received at least one prescribed drug to treat diabetes, migraine and/or depression during 2006 were identified through the Norwegian Prescription Database (NorPd) at the Norwegian Institute of Public Health $(15,16)$. This database contains information from all pharmacies on all prescribed drugs dispensed in Norway since 2004. For hospital pharmacies, information is available for out-patients only.

We included persons who had received minimum one prescription from the anatomical therapeutic chemical (ATC) classification system groups A10B (oral antidiabetic agents), N02CA (ergotamines), N02CC (triptans) and/or N06A (antidepressant agents) in 2006. Persons using clonidine (N02CX02) in monotherapy were not included, as clonidine has a number of other indications in addition to migraine, and seldom is recommended as first line treatment of migraine in monotherapy (17). We did not include persons receiving medications used in prophylactic treatment of migraine, such as antiepileptic agents, beta-adrenoceptor blockers, ACE-inhibitors and serotonin antagonists (pizotifen) unless they also required migraine agents from ATC-group N02CA or N02CC during 2006. Persons were included regardless of prescribed doses of the medications and no minimum doses or duration of use of any of the agents were required for inclusion.

The main explanatory variables in this cross-sectional study were the use of antidiabetic and antidepressant drug treatment, age and gender, while the main outcome was the use of migraine agents. In order to more easily compare our results with previous studies, we made the assumption that persons using oral antidiabetics, either in monotherapy or in combination with insulin, are expected to have type 2 diabetes, that persons using migraine agents likely have migraine, and that persons using antidepressant agents are expected to have a depressive disorder. The limitations of these assumptions are further described in the discussion section.

As a measure of one year prevalence, we estimated the number of persons with prescriptions according to age and gender for oral antidiabetic agents (used either in monotherapy or in combination with insulin), migraine agents and antidepressant agents. We then divided the study population according to whether they used antidepressant agents or not. Using logistic regression adjusting for sex and groups of age we estimated the overall OR for receiving migraine agents for persons using oral antidiabetic agents and antidepressant agents. Similarly, we estimated the overall OR for receiving migraine agents for persons using oral antidiabetic agents but no antidepressant agents. Further, we estimated age specific OR's adjusting for sex and also sex specific OR for the different age groups.

Although not a formal indication in Norway, metformin in monotherapy can be used in treating polycystic ovarian syndrome (PCOS). We therefore repeated the analyses excluding both men and women aged 20-39 years who received metformin in monotherapy.
Since we did not know how many of the women who were using metformin in monotherapy due to PCOS, we also excluded men with this treatment in order to compare the effect estimates between men and women for this age group.

The statistical analyses were performed using SPSS software version 18 (SPSS Inc., Chicago, IL, USA). As the database contains only age and gender in addition to the prescriptions, and since the individuals therefore were not traceable, it was not necessary to obtain permission from the National Authorities in Data and Information Security, or to have permission from the Regional Ethical Committee.

\section{RESUlTS}

In 2006 , a total of 5055 persons $(0.4 \%$ of the population aged 20-39 years) were prescribed oral antidiabetic agents, 26058 persons $(2.1 \%)$ used migraine agents, while 57424 persons $(4.6 \%)$ received antidepressant agents.

One year prevalence of prescriptions from all the 3 groups of medications increased from 20-29 to 30-39 years of age (figure 1). More than two times more women than men received oral antidiabetic agents (1 621 men and 3434 women), while almost four times more women than men received migraine agents (5 366 men and 20692 women). Women received approximately 1.5 times more antidepressant agents compared to men (22 458 men and 34966 women).

A total of 213 persons treated with oral antidiabetic agents also received migraine agents in 2006 (table 1). Of these, 160 persons did not use antidepressant agents, while 53 persons used antidepressant agents. Analysing only the persons not using antidepressant agents in both age-groups, we found that persons using oral antidiabetic agents had an overall increased prevalence of receiving migraine agents compared to persons not using oral antidiabetic agents $(\mathrm{OR}=1.48)$. For those who also used antidepressant agents the similar prevalence estimate was slightly lower $(\mathrm{OR}=1.37)$. For persons aged 20-29 years the corresponding prevalence estimate was actually higher for those using antidepressant agents compared to those who did not, but the numbers in this age group were small resulting in wide confidence intervals.

We found no major differences in prevalence rates between men and women (data not shown). Further, the sensitivity analysis excluding a total of 686 men and 2898 woman using metformin in monotherapy did not notably change the effect estimates (data not shown).

\section{DISCUSSION}

The well-known associations between depression and diabetes $(2,3)$ and between depression and migraine (11) were hypothesised to explain the previous finding of an increased use of migraine agents among persons 


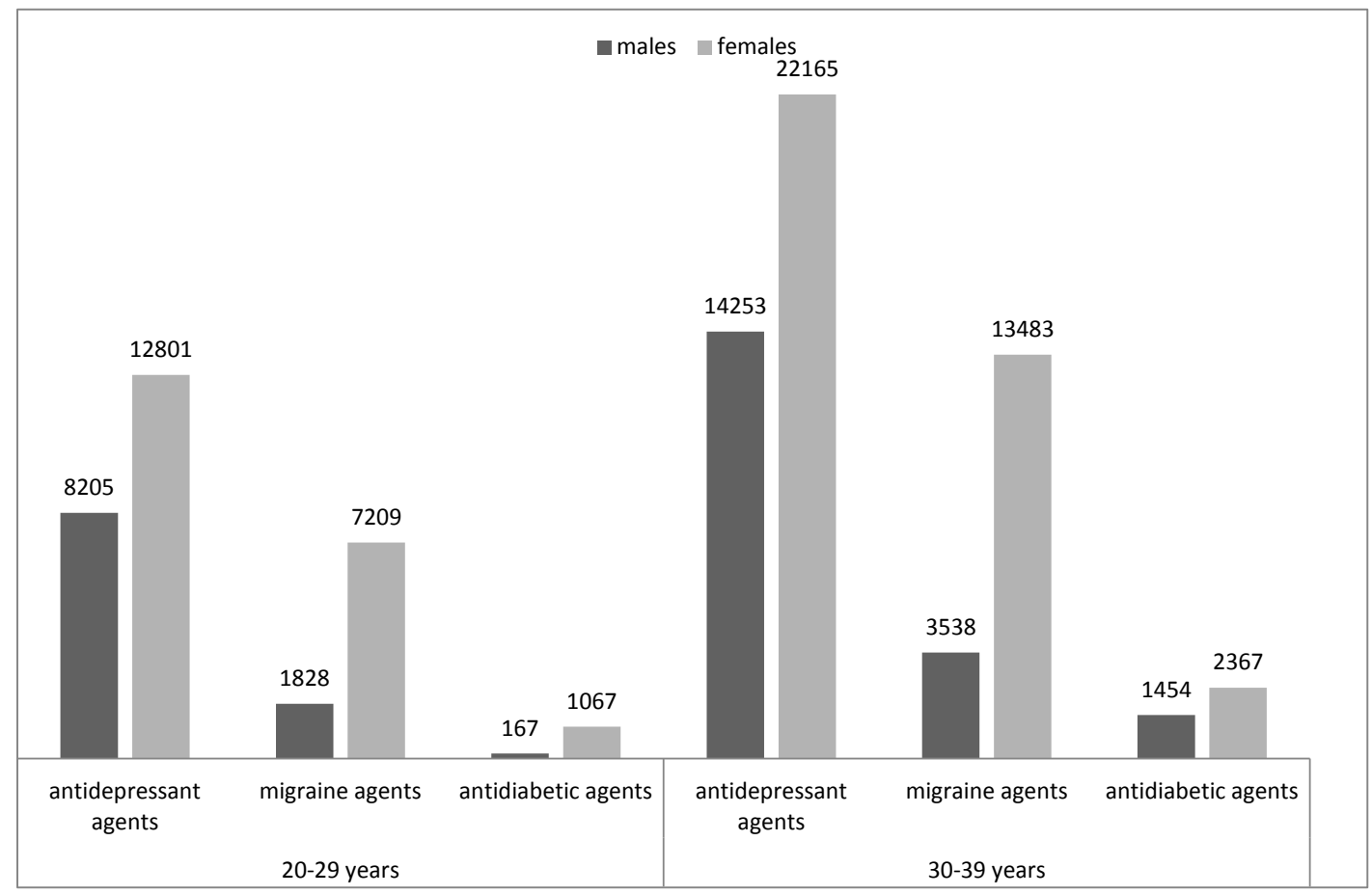

Figure 1. Prevalence of prescriptions according to age and sex for antidiabetic, migraine and antidepressant agents in the total Norwegian population in 2006.

Table 1. Use of migraine agents according to the use of oral anti-diabetic and antidepressant agents in the total Norwegian population in 2006.

\begin{tabular}{|c|c|c|c|c|c|c|c|}
\hline \multirow[b]{2}{*}{ Age } & & \multicolumn{3}{|c|}{ No use of antidepressant agents, $n=1197929$} & \multicolumn{3}{|c|}{ Use of antidepressant agents, $n=57424$} \\
\hline & & $\begin{array}{c}\text { Oral anti- } \\
\text { diabetic } \\
\text { agents, } n\end{array}$ & $\begin{array}{l}\text { Number of persons } \\
\text { receiving oral anti- } \\
\text { diabetic agents who } \\
\text { also receive migraine } \\
\text { agents, } \mathrm{n}(\%) \\
\end{array}$ & $\mathrm{OR}^{*}$ & $\begin{array}{c}\text { Oral anti- } \\
\text { diabetic } \\
\text { agents, } n\end{array}$ & $\begin{array}{l}\text { Number of persons } \\
\text { receiving oral anti- } \\
\text { diabetic agents who } \\
\text { also receive migraine } \\
\text { agents, } \mathrm{n}(\%)\end{array}$ & $\mathrm{OR}^{* *}$ \\
\hline \multirow[t]{2}{*}{$20-29$} & Yes & 1128 & $34(3.0)$ & \multirow[t]{2}{*}{$1.39(0.94,1.95)$} & 106 & $9(8.4)$ & \multirow[t]{2}{*}{$1.76(0.89,3.51)$} \\
\hline & No & 540685 & $8111(1.5)$ & & 20900 & $883(4.2)$ & \\
\hline \multirow[t]{2}{*}{$30-39$} & Yes & 3294 & $126(3.8)$ & \multirow[t]{2}{*}{$1.51(1.26,1.81)$} & 527 & $44(8.3)$ & \multirow[t]{2}{*}{$1.30(0.95,1.79)$} \\
\hline & No & 652822 & $14667(2.2)$ & & 35891 & $2184(6.1)$ & \\
\hline \multirow[t]{2}{*}{ Total } & Yes & 4422 & 160 & \multirow[t]{2}{*}{$1.48(1.26,1.74) * * *$} & 633 & 53 & \multirow[t]{2}{*}{$1.37(1.03,1.82) * * *$} \\
\hline & No & 1193507 & 22778 & & 56791 & 3067 & \\
\hline
\end{tabular}

*OR of receiving migraine agents when using oral antidiabetic agents but no antidepressant agents, adjusted for sex.

**OR of receiving migraine agents when using oral antidiabetic and antidepressant agents, adjusted for sex.

$* * *$ adjusted for sex and age group as a categorical variable.

using oral antidiabetic agents aged 20-39 years in a study covering the complete Norwegian population (13). The findings from the current study using the same study population and including data on the use of antidepressant agents showed that this hypothesis is not likely true. In age-group 20-39 years we found an increased use of migraine agents for patients using oral antidiabetic agents, both among those who used antidepressant agents and among those who did not.

Data on prescriptions is in this study used as a proxy for the prevalence of medically treated diabetes, migraine and depression in persons aged 20-39 years in the Norwegian population in 2006 , and we interpret the prevalence estimates as a measure of co-morbidity between the diseases. The validity of these measurements has been extensively discussed in two previous studies $(13,14)$. In brief, it is clear that many persons with diabetes, migraine or depression do not use medication from the ATC-groups included in this study. They will therefore be misclassified, underestimating the number of persons with the diseases. For example, the Norwegian Diabetes Association estimates that about $70 \%$ of patients with type 2 diabetes use antidiabetic agents (18). Nevertheless, our overall estimates for the prevalence of diabetes, migraine and depression in the total population are all comparable to well 
recognised studies on the prevalence of the diseases in Norway. For example, we found that $2.7 \%$ of the population aged 20 years and above used oral antidiabetic agents (14), while The Nord-Trøndelag Health (HUNT) 2 Survey undertaken in 1995-1997 reported the prevalence of type 2 diabetes in the corresponding age group to $2.6 \%$ (19). In addition, some of the medications included in this study for the treatment of migraine and depression have other indications. For instance, sumatriptan and ergotamine are also indicated in treatment of cluster headache, while some antidepressant agents also are indicated in treatment of chronic neuropathic pain, anxiety disorders, PTSD and bulimia. This reduces the specificity of the classification somewhat.

Our previous study showed an overall reduced prevalence of migraine in persons with diabetes (13). This "preventive" effect of diabetes on migraine was however only present among persons aged 50 years and above, while persons aged 40-49 years had the same prevalence of migraine as the non-diabetic population. The finding of an increased prevalence of migraine among persons less than 40 years indicates the presence of a strong age-related factor. The increased prevalence of co-morbid depression for persons with diabetes or migraine could obviously not explain this finding.

Other factors associated with an increased risk of diabetes in need of oral antidiabetic treatment, particularly among young persons, are overweight and obesity. Studies investigating whether overweight and obesity are associated with increased prevalence of migraine have, however, given conflicting results. A recent cross-sectional study found that obese have an increased prevalence of migraine in age group 20-55 years, (20), while a study from 2006 concluded that obesity and migraine did not occur as co-morbid conditions (21). Nevertheless, this study showed that attack fre- quency and severity of the migraine increased with increasing BMI. One study has investigated the effect of depression and anxiety on the relationship between obesity and migraine in a cross-sectional design, including data from over 700 migraineurs on BMI and psychiatric morbidity (22). They found that the prevalence of depression was highest among obese persons with migraine $(52 \%)$, and lowest in the normal weight group (40\%), and that obese persons with co-morbid depression were over 4 times more likely to have a higher headache frequency compared to normal weight migraineurs without depression.

The present study and our previous study on the association between diabetes and migraine were both cross-sectional, precluding a discussion on any direction on a possible causal association between the two diseases. Further, except for the use of antidepressant agents as a proxy for depression, we were not able to adjust our estimates for other possible confounding factors including socioeconomic status and life-style factors. Whether obesity is associated with an increased risk of migraine, or only related to migraine disability, this factor may have confounded our finding of an increased prevalence of migraine in young persons using oral antidiabetic agents. However, if our finding of an increased prevalence of migraine in young persons treated with oral antidiabetic agents irrespective of co-morbid depression is a result of a bias introduced by not being able to adjust the estimates for BMI, the use of antidepressant agents must be associated with BMI in young persons using oral antidiabetica. We find this less likely.

In conclusion, the findings in the present study give no support for the hypothesis that the increased prevalence of migraine in young persons treated with oral antidiabetic agents partly could be explained by comorbid depression.

\section{REFERENCES}

1. IDF Diabetes Atlas, $5^{\text {th }}$ edition. International Diabetes Federation, 2011: 18-19.

2. Ali S, Stone M, Peters J, Davies M, Khunti K. The prevalence of co-morbid depression in adults with Type 2 diabetes: a systematic review and meta-analysis. Diabet Med 2006; 23: 1165-73.

3. Mezuk B, Eaton W, Albrecht S, Golden SH. Depression and type 2 diabetes over the lifespan. A meta-analysis. Diabetes Care 2008; 31: 2383-2390.

4. Moussavi S, Chatterji S, Verdes E, Tandon A, Patel V, Ustun B. Depression, chronic diseases, and decrements in health: results from the World Health Surveys. Lancet 2007; 370: 851-858.

5. Ciechanowski P, Katon W, Russo J. Depression and diabetes: impact of depressive symptoms on adherence, function and costs. Arch Intern Med 2000; 160: 3278-3285.

6. Richardson L, Egede K, Mueller M, Echols C, Gegregziabher M. Longitudinal effects of depression on glycaemic control in veterans with Type 2 diabetes. Gen Hosp Psychiatry 2008; 30: 509-514.

7. Black S, Markides K, Ray L. Depression predicts increased incidence of adverse health outcomes in older Mexican Americans with type 2 diabetes. Diabetes Care 2003; 26: 2822-2828.

8. Ismail K, Winkley K, Stahl D, Chalder T, Edmonds M. A cohort Study of people with diabetes and their first foot ulcer: the role of depression on mortality. Diabetes Care 2007; 30: 1473-1479.

9. Zhang X, Norris S, Gregg E, Chang Y, Beckles G, Kahn H. Depressive symptoms and mortality among persons with and without diabetes. Am J Epidemiol 2005; 161: 652-660.

10. Executive Summary: Standards of Medical Care in Diabetes. Diabetes Care 2011; 34 (Suppl 1). 
11. Hamelsky SW, Lipton RB. Psychiatric comorbidity of migraine. Headache 2006; 46: 1327-1333.

12. Aamodt AH, Stovner LJ, Midthjell K, Hagen K, Zwart J-A. Headache prevalence related to diabetes mellitus. The Head-HUNT Study. Eur J Neurol 2007; 14: 738-744.

13. Berge LI, Riise T, Fasmer OB, Hundal Ø, Oedegaard KJ, Midthjell K, Lund A. Does diabetes have a protective effect on migraine? Epidemiology 2013; 24: 129-134.

14. Berge LI, Riise T, Fasmer OB, Lund A, Oedegaard KJ, Hundal Ø. Risk of depression in diabetes is highest for young persons using oral anti-diabetic agents. Diabet Med 2012; 29: 509-514.

15. Furu K. Establishment of the nation wise Norwegian Prescription Database (NorPD) - new opportunities for research in pharmacoepidemiology in Norway. Norsk Epidemiologi 2008; 18: 125-136.

16. Norwegian national register of prescriptions (NorPd). http://www.reseptregisteret.no. Accessed 15.10.2009.

17. Goadsby P, Sprenger T. Current practice and future directions in the prevention and acute management of migraine. Lancet Neurol 2010; 9: 285-298.

18. http://www.diabetes.no/Type+2-diabetes.9UFRnQ4P.ips. Accessed 04.05.2012.

19. Midthjell K, Krüger Ø, Holmen J, et al. Rapid changes in the prevalence of obesity and known diabetes in an adult Norwegian population. The Nord-Trøndelag Health Surveys: 1984-1986 and 1995-1997. Diabetes Care 1999; 22:1813-1820.

20. Peterlin BL, Rosso AL, Rapport AM, Scher AI. Obesity and migraine: The effect of age, gender and adipose tissue distribution. Headache 2010; 50: 52-62.

21. Bigal ME, Liberman JN, Lipton RB. Obesity and migraine: a population study. Neurology 2006; 66: 545-550.

22. Tietjen G, Lee Peterlin B, Brandes JL, et al. Depression and anxiety: Effect on the migraine-obesity relationship. Headache 2007; 47: 866-875. 